\title{
ПЕРИНАТАЛЬНЫЙ МЕНЕДЖМЕНТ ПРИ ЗАБОЛЕВАНИЯХ ЩИТОВИДНОЙ ЖЕЛЕЗЫ: СИНОПСИС И АЛГОРИТМЫ
}

\begin{abstract}
—еринатальный менеджмент при заболеваниI ях щитовидной железы (ЩЖ) представляет собой уникальный шанс для командного подхода, интеграции знаний и клинических рекомендаций. На сегодняшний день в связи с постоянным совершенствованием методик фармакотерапии и хирургических методов лечения у многих женщин с патологией ЩЖпоявились возможности вынашивать беременность и родить здорового ребенка.

В данной публикации авторы приводят результаты современной акушерской тиреоидологии.
\end{abstract}

\section{ФАКТОРЫ, СТИМУЛИРУЮЩИЕ ЩЖ ПРИ БЕРЕМЕННОСТИ}

К данным факторам относятся:

(3) повышение концентрации тироксинсвязывающего глобулина (вторичный эффект воздействия эстрогенов на ткани печени);

(5) повышение уровня хорионического гонадотропина, действующего на рецепторы к тиреотропному гормону (ТТГ);

5 обеспечение плода йодом, находящимся в щЖ матери.

Согласно рекомендациям Американской тиреоидной ассоциации (АТА) по обеспечению йодом, беременная должна получать йод в дозе 229 мкг/сут, а кормящая женщина 289 мкг/сут, из которых 150 мкг она должна получать из витаминного комплекса.

Нормальная функция ЩЖ обеспечивает повышение синтеза тиронинов при беременности и поддержку их уровня в течение всего периода гестации.

Активный синтез тиронинов ЩЖ матери происходит к 18-й неделе беременности, поглощение йода фолликулами щЖ - с 10-й по 14-ю неделю. Активный трансфер материнского тироксина к плоду происходит с ранних сроков беременности: рецепторы к тироксину в головном мозге плода определяются уже в 8 нед, поэтому даже умеренный дефицит материнских тиронинов в I триметре беременности может привести к поражению ЦНС у детей, проявляющемуся в отсроченном периоде снижением когнитивных функций и задержкой психоневрологического развития.

Основные биомаркеры оценки функции ЩЖ и предикторы возможных осложнений при патологии ЩЖ у матери (схема 1):

(5TГ-тест первого уровня;

(3) определение уровня антител к тиреопероксидазе (АТ-ТПО);

3 определение медианы йодурии (в регионах, признанных йододефицитными); о определение уровня антител к рецептору тканей щЖ (TRA)

\section{ПРЕКОНЦЕПЦИОННОЕ КОНСУЛЬТИРОВАНИЕ}

Основные проблемы, связанные с патологией щЖ, требующие консультирования до беременности:

1. Предсуществующий гипертиреоз: основная задача - добиться эутиреоидного состояния на момент наступления беременности путем: д длительного применения тиреостатиков;

s использования радиойодной абляции;

(3) предельно тотальной тиреоидэктомии.

2. Получение комбинированной терапии при карциноме ЩЖ:

(5) прменение контрацепции в течение 6-12 мес после получения лечебной дозы радиойода;

(3) адаптация дозы экзогенного левотироксина в зависимости от лабораторных показателей, удерживать уровень ТТГ необходимо на низко-нормальном уровне, но следует избегать клинического гипертиреоза.

3. Предсуществующий гипотиреоз:

(5) необходимо рекомендовать повышение дозы экзогенного левотироксина вскоре после зачатия, адаптировать его дозы по отношению к увеличению срока беременности;

ร у женщин, получавших заместительную гормональную терапию левотироксином, с уровнем ТТГ перед зачатием более 1,3 мЕд/л в 58\% случаев возникает необходимость повышения дозы левотироксина в I триместре беременности, а у лиц с уровнем ТТГ менее 1,3 мЕд/л подобная ситуация возникает в 17\% случаев;

3 пациенткам, получающим заместительную гормональную терапию левотироксином, необходимо рекомендовать прием сульфата железа и препаратов кальция через 2 ч от момента приема левотироксина.

\section{ФУНКЦИЯ СИСТЕМЫ \\ МАТЬ-ПЛАЦЕНТА-ПЛОД \\ ПРИ ЗАБОЛЕВАНИЯХ ЩЖ}

Проникновение материнского гормона и лекарственного гормонального препарата в плаценту при наличии заболеваний щЖ у женщин представлено в таблице 1.

\section{ГЕСТАЦИОННЫЙ ГИПЕРТИРЕОЗ}

Дифференциальный диагноз болезни Грейвса и гестационного тиреотоксикоза указан в таблице 2 .

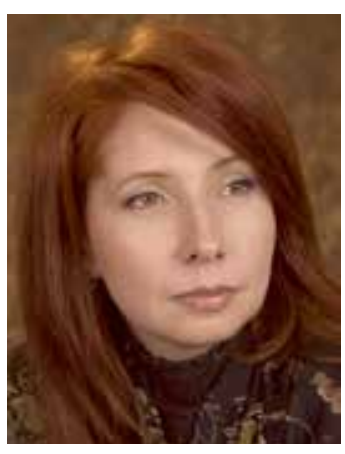

Ю.В. ДАВЫДОВА д.мед.н., зав. отделением акушерских проблем

экстрагенитальной патологии Института педиатрии, акушерства и гинекологии НАМН Украинь

К.Г. АПРЕСОВА

д.мед.н., главный научный сотрудник отделения акушерских проблем экстрагенитальной патологии Института педиатрии, акушерства и гинекологии НАМН Украины

И.В. ЧИБИСОВА

начальник отдела акушерско-

гинекологической помощи

Департамента материнства, детства и санаторного обеспечения МЗ Украины, Институт педиатрии, акушерства и гинекологии НАМН Украины

А.О. ОГОРОДНИК

к.мед.н., младший научный сотрудник отделения акушерских проблем экстрагенитальной патологии Института педиатрии акушерства и гинекологии НАМН Украинь

М.П. дВулит

к.мед.н., старший научный сотрудник отделения акушерских проблем экстрагенитальной патологии Института педиатрии, акушерства и гинекологии НАМН Украины

Л.М. БУлИк

к.мед.н., Институт педиатрии, акушерства и гинекологии НАМН Украины 


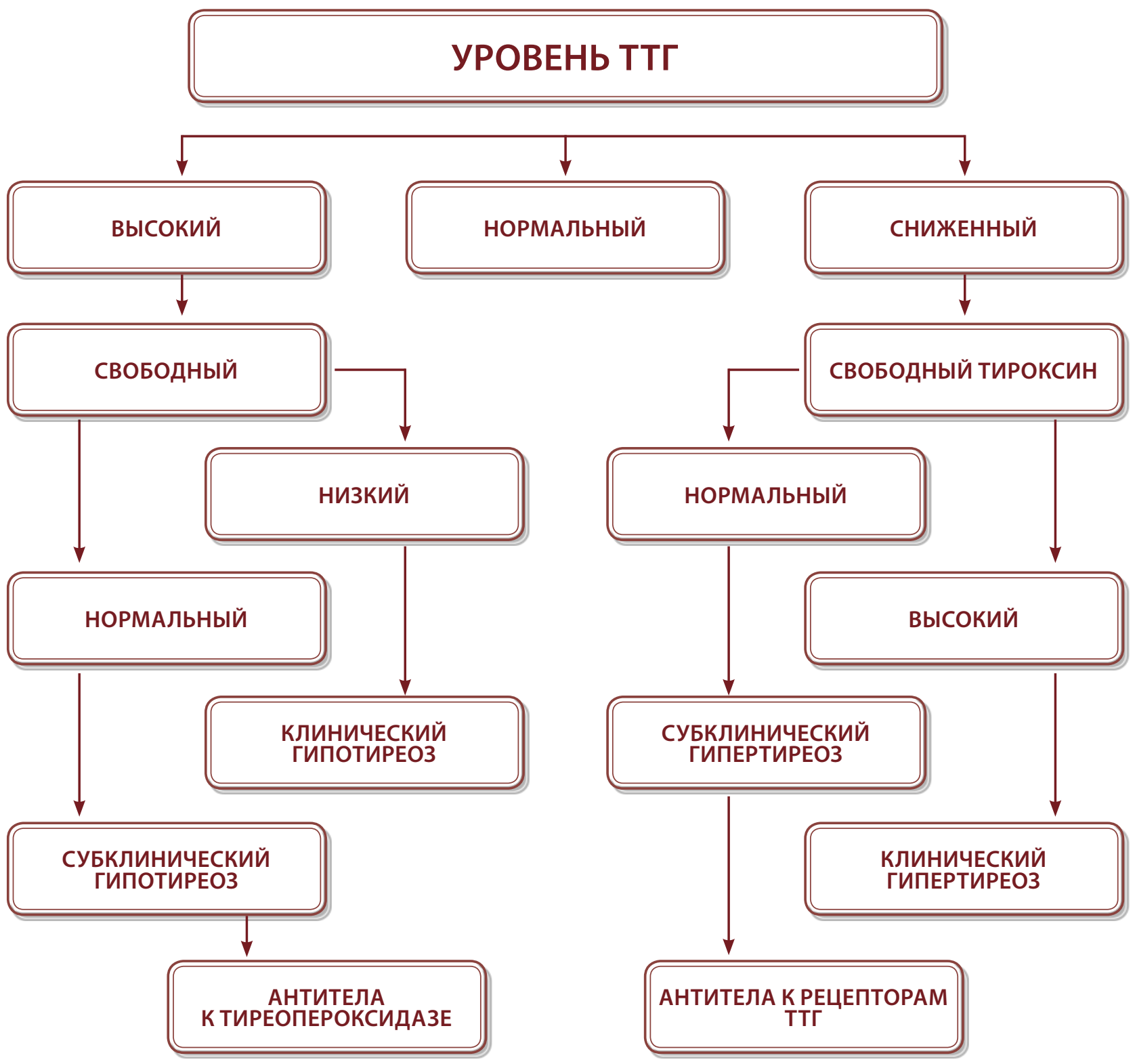

CXEMA 1.

АЛГОРИТМ ОЦЕНКИ ФУНКЦИИ ЩЖ ПО УРОВНю ТТГ

ТАБЛИЦА 1. ПРОНИКНОВЕНИЕ В ПЛАЦЕНТУ ГОРМОНОВ И ЛЕКАРСТВЕННЫХ СРЕДСТВ

Гормон матери или медицинский препарат, который она получает

Тиреотропный рилизинг-гормон

TTГ

Тироксин (Т4)

Метимизол

Пропилтиоурацил

Карбамазол
Проникновение в плаценту

Проникает

Не проникает

Проникает, особенно важно в I триместре

$$
\begin{aligned}
& \text { Проникает } \\
& \text { Проникает } \\
& \text { Проникает }
\end{aligned}
$$

ТАБЛИЦА 2. ДИФФЕРЕНЦИАЛЬНЫЙ ДИАГНОЗ БОЛЕЗНИ ГРЕЙВСА И ГЕСТАЦИОННОГО ТИРЕОТОКСИКОЗА

\begin{tabular}{|c|c|c|}
\hline Симптомы & Болезнь Грейвса & Гестационный гипертиреоз \\
\hline Симптомы до беременности & + & - \\
\hline Симптомы во время беременности & ++ & $-/+$ \\
\hline Тошнота, рвота & $-1++$ & - \\
\hline 306, офтальмопатия & + & - \\
\hline АТ-ТПО/ТRАЬ & + & Норма \\
\hline Результаты УЗИ & Повышенная васкуляризация & - \\
\hline
\end{tabular}




\title{
ТАБЛИЦА З. ПОТЕНЦИАЛЬНЫЕ МАТЕРИНСКИЕ И ПЕРИНАТАЛЬНЫЕ ОСЛОЖНЕНИЯ ПРИ БОЛЕЗНИ ГРЕИВСА У МАТЕРИ
}

Невынашивание

Гипертензивные осложнения при беременности

Преждевременные роды

Сердечная недостаточность

Тиреоидный шторм

Преждевременная отслойка нормально расположенной плаценты

Инфицирование

\author{
Перинатальные осложнения \\ Низкая масса тела при рождении: \\ 5 недоношенность \\ 9 малый вес для гестационного возраста \\ 5 задержка внутриутробного развития \\ Мертворождение \\ Тиреоидная дисфункция: \\ 3 фетальный гипертиреоз \\ 5 фетальный гипотиреоз \\ s. неонатальный гипертиреоз \\ 5 неонатальный 306 \\ s. неонатальный центральный гипотиреоз
}

Потенциальные материнские и перинатальные осложнения при болезни Грейвса у матери представлены в таблице 3.

ATA рекомендует прием в среднем 20 мг/сут метимизола или 100-150 мг пропилтиоурацила 3 раза вдень, что позволяет достичь терапевтического эффекта у большинства женщин. При этом лабораторным ориентиром служит уровень свободного тироксина на верхней границе нормы. Клинический эффект возникает через 2-6 нед от момента старта терапии, изменения лабораторных тестов происходят через 2 нед, достижение эутиреоза на фоне приема медикаментов - через 5-7 нед. При минимальных симптомах гипертиреоза старт
Субклинический гипотиреоз - повышенный уровень ТТГ и нормальный уровень свободного Т4.

Рекомендованные нормальные уровни ТТГ при беременности - 2,5 мЕд/л в I триместре и 3,0 мЕд/л во II и III триместрах.

Клинический гипотиреоз - повышенный уровень ТТГ и сниженный уровень свободного Т4.

До сих пор спорным остается вопрос о том, какая тактика наиболее предпочтительна: универсальный скрининг функции ЩЖ всем женщинам детородного возраста перед планированием беременности или скрининг в отдельных группах женщин.

ТАБЛИЦА 4. ПОБОЧНЫЕ ЭФФЕКТЫ МЕТИМИЗОЛА И ПРОПИЛТИОУРАЦИЛА

\begin{tabular}{|c|c|}
\hline Метимизол & Пропилтиоурацил \\
\hline $\begin{array}{l}\text { Кожный зуд } \\
\text { Прурит } \\
\text { Мигрирующий полиартрит } \\
\text { Псевдоволчаночный синдром } \\
\text { Холестатическая желтуха } \\
\text { Агранулоцитоз } \\
\text { Метимизоловая эмбриопатия: } \\
\text { атрезия хоан и атрезия пищевода } \\
\text { арlasia сиtis } \\
\text { нарушения слуха } \\
\text { дисморфизм лица } \\
\text { задержка развития }\end{array}$ & $\begin{array}{l}\text { Кожный зуд } \\
\text { Прурит } \\
\text { Мигрирующий полиартрит } \\
\text { Псевдоволчаночный синдром } \\
\text { Пропилтиоурациловая гепатотоксичность }\end{array}$ \\
\hline
\end{tabular}

терапии можно начинать с 10 мг/сут метимизола и 50 мг пропилтиоурацила 2-3 раза в день. При достижении клинического улучшения состояния (набор массы тела и исчезновение тахикардии) дозу медикаментов следует снизить вдвое. Также необходимо принять во внимание побочные эффекты тиреостатической терапии (табл. 4).

В среднем 4000 американок получают тиреостатики, преимущественно пропилтиоурацил, при этом в среднем у четырех из них могут развиться тяжелые осложнения, связанные с приемом данного препарата. Исходя из вышеизложенных осложнений, АТА и Управление по контролю за пищевыми продуктами и лекарственными средствами (FDA) рекомендуют прием пропилтиоурацила в I триместре беременности и метимизол - во II триместре.

Грудное вскармливание разрешено, если дневная доза пропилтиоурацила и метимизола ниже 300 и 20 мг/сут соответственно.

\section{ГИПОТИРЕОЗ}

Двумя наиболее частыми причинами предсуществующего гипотиреоза являются аутоиммунный тиреоидит (зоб Хашимото) и гипотиреоз, связанный с радиойодной абляцией ЩЖ или ее хирургическим удалением.
Рекомендации АТА относительно показаний к селективному скринингу функции ЩЖ при беременности:

3 заболевание ЩЖ в анамнезе или оперативное вмешательство на щЖ;

возраст старше 50 лет;

(5) симптомы дисфункции ЩЖ или наличие зоба;

3. наличие АТ-ТПО;

s сахарный диабет 1-го типа или другие аутоиммунные заболевания;

с семейный анамнез патологии щЖ;

s ожирение;

( прием амиодарона и препаратов лития, недавнее введение йодсодержащего радиоконтраста;

s вспомогательные репродуктивные технологии, примененные перед данной беременностью;

3 проживание в йоддефицитных регионах.

Рекомендации по лечению:

1. Старт терапии, как только установлен диагноз гипотиреоза.

2. Если диагноз впервые установлен во время беременности, начальная доза левотироксина должна составить100 мкг/сут с последующим лабораторным тестированием. 
3. Доза может быть рассчитана от веса пациентки (2,0-2,4 мкг/кг массы тела) при беременности.

3 До беременности дозу левотироксина рассчитывают как 1,7-2,0 мкг/кг массы тела.

5. Лабораторные тесты - нормализация через 2 нед. Тестирование ТТГ следует проводить каждые 2-6 нед. течение 6-12 мес после последней процедуры. При этом необходимо помнить, что у них может нарушиться менструальный цикл или могут возникнуть проблемы с фертильностью, повышенный риск невынашивания беременности и врожденной патологии, если этот срок не будет соблюден. Женщин, получивших высокие дозы радиойод-

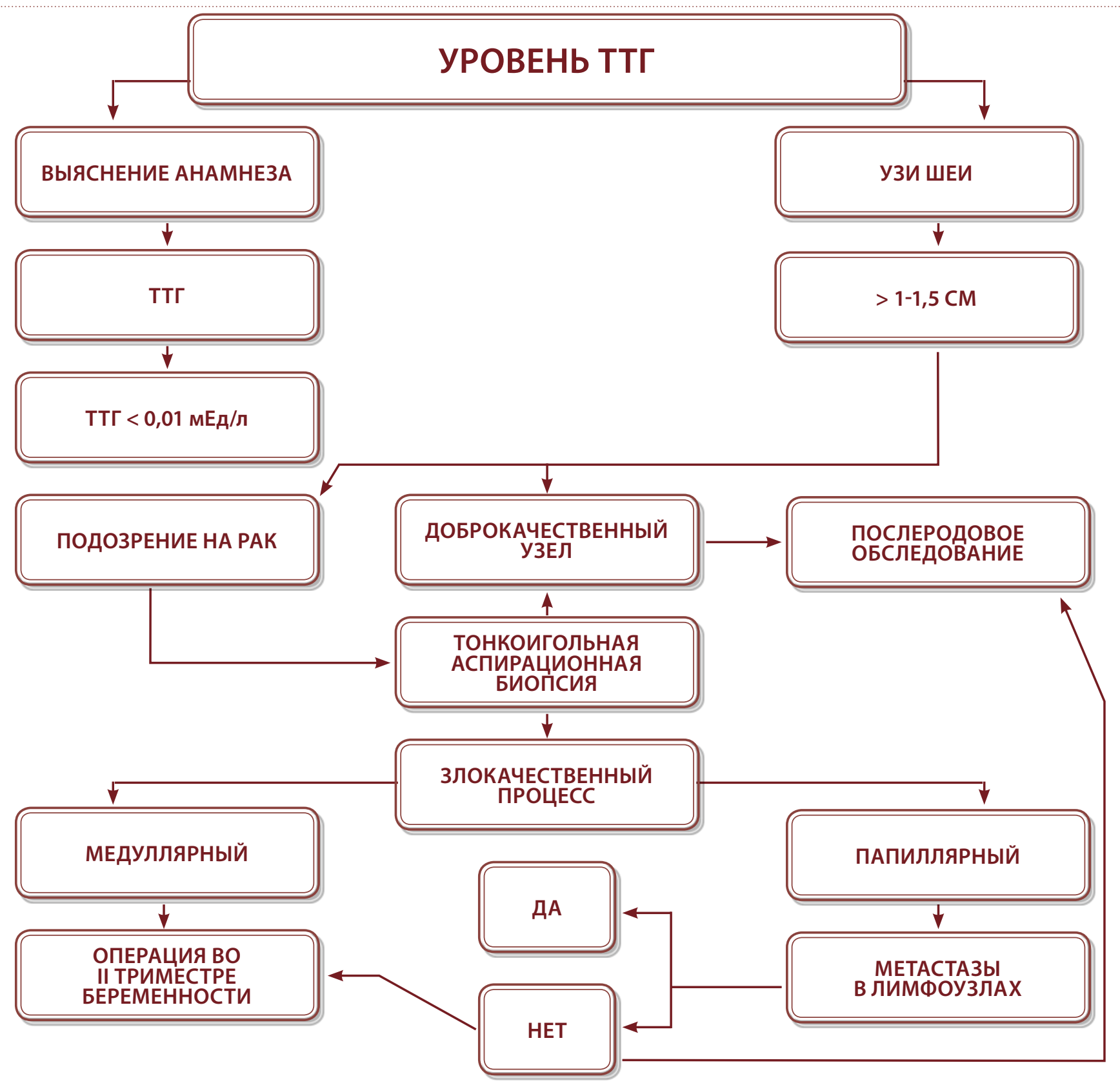

CXEMA 2.

ТАКТИКА ВЕДЕНИЯ ПАЦИЕНТКИПРИПАЛЬПИРУЕМЫХ УЗЛАХ ЩЖ

\section{РАК ЩЖ ПОСЛЕ КОМБИНИРОВАННОЙ}

\section{ТЕРАПИИ ДО БЕРЕМЕННОСТИ}

Если женщине проведено комбинированное лечение рака ЩЖ, включающее тотальную тиреоидэктомию, радиойодсканирование (радиойодтерапию), супрессивную терапию левотироксином, то при папиллярной или фолликулярной морфологии опухоли сегодня нет оснований рассматривать беременность как фактор риска рецидива заболевания.

Женщинам, получившим радиойодтерапию, необходимо воздерживаться от планирования беременности в ной абляции, необходимо информировать о том, что для них существует высокий риск преждевременного истощения функции яичников, более ранней менопаузы.

При наступлении беременности ориентир для проведения терапии левотироксином составляет 0,1-0,5 мЕд/л.

В последние 20 лет в 2,4 раза увеличилось число небольших папиллярных карцином, определяемых при беременности (3,6 случаев на 100000 в 1975 г. и 8,7 - на 100000 в 2002 г.). В частности, это объясняется рутинным проведением УзИ ЩЖ при беременности. В 75-80\% случаев определяются папиллярные карциномы, в 15-20\% - 
фолликулярные, в возрасте до 50 лет крайне редко встречаются медуллярные и низкодифференцированные опухоли.

\section{УЗЛОВОЙ ЗОБ ПРИ БЕРЕМЕННОСТИ}

Клинически определяемый узловой зоб при беременности диагностируется у $10 \%$ женщин (схема 2).

Риск того, что узел в ЩЖ будет злокачественным, составляет 5-10\% в зависимости от наличия факторов риска. Алгоритм обследования включает:

s пальпацию образования;

与 Узи щЖ;

(3) тонкоигольную аспирационную биопсию узла;

s верификацию характера опухоли.

АТА рекомендует следующий комплекс мероприятий:

1. Если размеры солидного узла < 1-1,5 см, показано углубленное обследование в послеродовом периоде.

2. Узлы размерами > 1-1,5см должны быть обязательно пунктированы путем тонкоигольной аспирационной биопсии.

3. Немедленное хирургическое вмешательство показано при обструкции трахеи.

4. Если диагностирован злокачественный или с подозрением на злокачественный узел, отложить хирургиче- ское вмешательство возможно только при отсутствии метастазов в лимфоузлы или больших размеров повреждения, а также если не отмечено вовлечение лимфоузлов в медуллярный рак.

5. При злокачественном процессе или быстро растущем узле оперативное вмешательство должно быть проведено во ІІ триместре беременности.

6. При фолликулярной и папиллярной карциноме можно рассматривать вопрос о перенесении оперативного вмешательства на послеродовой период, так как при этой патологии не отмечается быстрого прогресса опухолевого процесса.

\section{ПОСЛЕРОДОВОЙ ТИРЕОИДИТ}

Послеродовой тиреоидит (ПРТ) - транзиторное нарушение функции ЩЖ в течение первого года после родов у женщин, у которых до беременности установлено эутиреоидное состояние. У $27 \%$ женщин с ПРТ в начале беременности установлен повышенный уровень антитиреоидных антител. У 2-21\% пациенток с ПРТ через 12 мес диагностируется гипотиреоз. В данной группе женщин рекомендуется прием селена. Так, в группе женщин, получавших 2 мкг/сут селена во время беременности, ПРТ развивался в $28,6 \%$ случаев, а в группе получавших плацебо - в 48,6\%.
КЛЮЧЕВЫЕ ПОЗИЦИИ

В I триместре беременности необходимо дифференцировать болезнь Грейвса от гестационного тиреотоксикоза.

Недавние исследования доказали наличие риска печеночной недостаточности при приеме тиреостатиков (пропилтиоурацила), поэтому рекомендовано назначать пропилтиоурацил в I триместре беременности, а начиная с 13 нед метимизол.

Доза антитиреоидных препаратов должна быть адаптирована в индивидуальном порядке каждой беременности, наилучший результат оказывает минимальная доза препарата, позволяющая удерживать уровень свободного тироксина на верхней границе нормы.
Грудное вскармливание не рекомендуется женщинам, получающим больше 20 мг метимизола или 200 мг пропилтиоурацила в суточной дозе.

У женщин, имеющих факторы риска заболевания ЩЖ, необходимо провести оценку функции ЩЖ как можно раньше в I триместре беременности (предпочтительно в преконцепционном

периоде). Таким женщинам рекомендуется определять уровень ТТГ, свободного Т4, АТ-ТПО.

Послеродовой тиреоидит поражает 16,7\% всех женщин в послеродовом периоде. Длительные наблюдения в такой группе женщин показали высокий риск развития постоянного гипотиреоза через 5-10 лет.

\section{ЛИТЕРАТУРА}

\section{Abbassi-Ghanavati M. et al.}

Pregnancy outcomes in women with thyroid peroxidase antibodies. Obstet Gynecol. 116: 381, 2010.

2. American Thyroid Association.

Statement on early maternal thyroid recognition? Clinical management and research direction. Thyroid. 15: 77, 2005.

\section{Krassas E.G., Poppe K., Glinoer D.}

Thyroid function and human reproductive health. Endocr. Rev. 20: 762, 2010. 4. Vannucchi D., Perino M., Rossi S. et al.

Clinical and molecular features of thyroid cancer diagnosed during pregnancy. Euro J. Endocrinol. 162: 145, 2010. 